\title{
WEAK GALAXY-GALAXY LENSING IN HST DATA
}

\author{
TIM EBBELS ${ }^{1}$, JEAN-PAUL KNEIB ${ }^{2}$ \& RICHARD ELLIS ${ }^{1}$ \\ ${ }^{1}$ Institute of Astronomy, Madingley Road, Cambridge, \\ CB3 OHA, U.K. \\ ${ }^{2}$ Observatoire Midi-Pyrenees, 14 Av. E.Belin, 31400 Toulouse, \\ France
}

\section{Description}

We expand on the work of Brainerd, Blandford \& Smail (1996) (BBS), using a larger archival WFPC-2 dataset including many galaxy redshifts. It is clear from our data that the ellipticity distribution of images changes substantially with observed magnitude (figure 1 , left) which is shown by simulations to be mainly the result of detection effects. We have detected a lensing signal, using a similar selection method to BBS and are also implementing a maximum likelihood method to constrain halo parameters. Using simulations, we show in figure 1 (right) that the signal is consistant with typical halo velocity dispersions of $\sigma^{*} \sim 70-100 \mathrm{kms}^{-1}$ However, the radial extent of the halos is less well constrained.
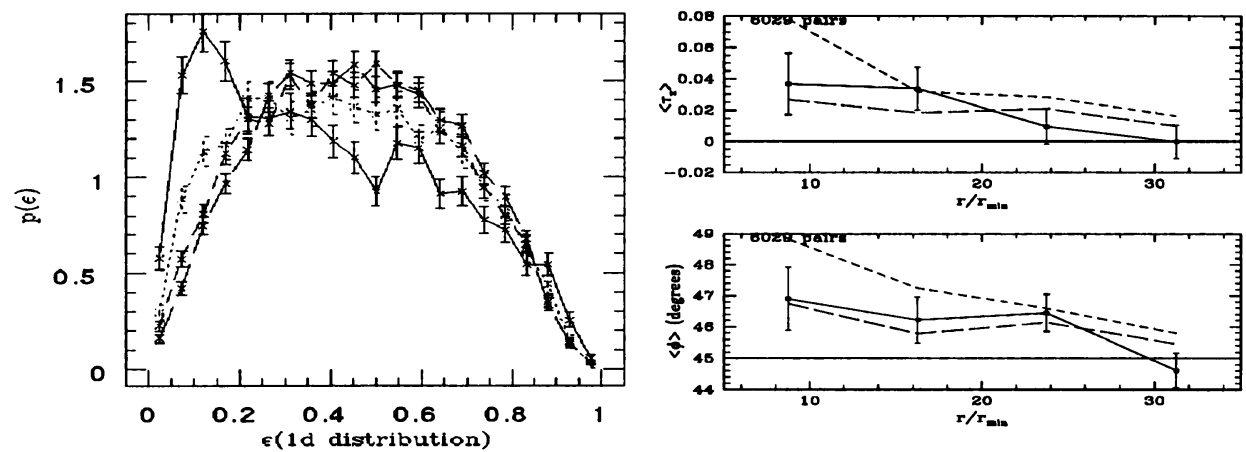

Figure 1. left: Change in the ellipticity distribution with magnitude (solid, dotted, dashed \& long dashed lines: $I=19-23,23-24,24-25,25-26$. right. Lensing signal from data (solid line) and simulations using $\sigma *=100 \& 70 \mathrm{kms}^{-1}$ (short \& long dashes)

\section{References}

Brainerd, T.G., Blandgord, R.D. \& Smail, I., 1996, ApJ., 466, 623. 